\title{
Seducidos y abandonados: el aislamiento social de los pobres urbanos
}

\section{Rubén Kaztman}

Director del Programa

de Investigación

sobre Integración, Pobreza

y Exclusión Social,

Universidad Católica

de Uruguay

kaztman@adinet.com.uy
Este artículo examina los efectos que han tenido algunas trans-

formaciones recientes en la estructura social de aquellos países latinoamericanos de temprano desarrollo, sobre el aislamiento social de los pobres urbanos. Esas transformaciones se refieren principalmente a los mercados de trabajo y a ciertas estructuras de oportunidades que son fuente de formación de recursos humanos y de capital social. Se argumenta aquí que, como resultado de esas transformaciones, se debilitan los vínculos de los pobres urbanos con el mercado de trabajo y se estrechan los ámbitos de sociabilidad informal con personas de otras clases sociales, lo que conduciría a su progresivo aislamiento. Se analiza asimismo la reducción de oportunidades para acumular capital social individual, capital social colectivo y capital cívico, y se examinan las características particulares que asumen los procesos de segregación residencial en las grandes ciudades de los países considerados. 


\section{I}

\section{Introducción}

La mayoría de las políticas públicas que se llevan a cabo en los países de la región para elevar el bienestar de los pobres urbanos han descuidado los problemas de su integración en la sociedad, operando como si el solo mejoramiento de sus condiciones de vida los habilitara para establecer (o restablecer) vínculos significativos con el resto de su comunidad. Sólo en los últimos años, y a medida que se comprobaba la agudización de los problemas de segmentación social que acompañan el despliegue de los nuevos modelos de crecimiento, el discurso de académicos y de encargados de políticas sociales comenzó a reflejar una preocupación por los problemas de aislamiento social de los pobres urbanos y por los mecanismos que nutren y sostienen esas situaciones, más allá de la consideración de sus apremios económicos y de sus carencias específicas. En efecto, la incorporación en el léxico especializado de las nociones de exclusión, desafiliación, desvalidación, fragmentación y otras semejantes revela la inquietud por la creciente proporción de población que, además de estar precaria e inestablemente ligada al mercado de trabajo, se ve progresivamente aislada de las corrientes predominantes (mainstream) ${ }^{1}$ en la sociedad. Este fenómeno, cualquiera sea el término que se le aplique, implica vínculos frágiles $-\mathrm{y}$ en último extremo inexistentes- entre los pobres urbanos y las personas e instituciones que orientan su desempeño por las normas y valores dominantes en la sociedad en un determinado momento histórico.

Una virtud de estos enfoques es la incorporación de la estructura social como elemento explícito del

$\square$ Este artículo es una versión revisada de un documento presentado en el IV Foro Internacional organizado por el Centro de Análisis y Difusión de Economía Paraguaya (CADEP) en Asunción, el 23 y 24 de noviembre de 2000. La sección ha sido extractada de Kaztman (2001); estudio financiado por SIEMPRO/UNESCO en el marco del Observatorio Social. El autor agradece a Raúl Bissio y Eduardo de León por sus excelentes comentarios y sugerencias a versiones previas de este artículo.

${ }^{1}$ La expresión "corrientes predominantes" (mainstream) se refiere al sector de la sociedad cuyas aspiraciones de integración y movilidad social se canalizan a través de vías institucionales, y cuyos comportamientos y expectativas se ajustan a las normas y valores predominantes. La palabra "predominante" suele denotar no sólo el poder y el prestigio de este sector $y$, por ende, su capacidad de difundir normas, valores y modelos de comportamiento, sino también su peso numérico dentro de la sociedad. marco conceptual con que se interpretan los fenómenos de pobreza. La localización de los pobres dentro de esa estructura varía no sólo según la profundidad de las brechas que los separan de otras categorías sociales en el mercado de trabajo, sino también según el grado de segmentación en cuanto a la calidad de los servicios de todo tipo y el grado de segregación residencial. Estas consideraciones permiten ampliar el campo de comprensión de los fenómenos de pobreza más allá de los esquemas que la conciben como producto de las vicisitudes de la economía, o como resultado del portafolio de recursos de los hogares y de su capacidad de movilizarlos de manera eficiente; al mismo tiempo, abren expectativas acerca de la posibilidad de formular políticas que atiendan dichos fenómenos en forma más integral que en el pasado.

En las notas que siguen presentaré algunas hipótesis sobre la naturaleza y los factores determinantes del aislamiento social de los pobres urbanos, con la esperanza de que los resultados de su puesta a prueba contribuyan a mejorar la eficacia de los programas contra la pobreza. De más está decir que, dado que todavía son muy escasas las investigaciones sobre estas materias en los países de la región, la mayoría de dichas hipótesis se encuentran en estado embrionario. ${ }^{2}$

Entre los factores que más poderosamente inciden en los cambios que experimenta la pobreza urbana en los países de la región se encuentran las transformaciones que ocurren en los mercados de trabajo. Bajo el impulso de procesos de desindustrialización, achicamiento del Estado y acelerada incorporación de innovaciones tecnológicas en algunas áreas de actividad, se reduce la proporción de ocupaciones protegidas y estables, aumentan las disparidades de ingreso entre trabajadores de alta y de baja calificación, y se intensifican los problemas de desempleo y subempleo, que afectan en particular a estos últimos. ${ }^{3} \mathrm{~A}$ menos que

\footnotetext{
${ }^{2}$ Algunos resultados de estudios realizados en Argentina y Uruguay se encuentran en Kaztman (1997), Kaztman (coord., 1999) y Kaztman y otros (1998).

${ }^{3}$ Por supuesto, estas relaciones no deben interpretarse de manera mecánica, habida cuenta de que los Estados muestran distintas disposiciones y capacidades -que en general suelen traducir valores colectivos enraizados en las matrices institucionales de cada paíspara amortiguar los impactos de las transformaciones económicas sobre la pobreza y la desigualdad. Pese a ello, no se puede desco-
} 
existan políticas específicamente diseñadas para prevenir que ello suceda, la ampliación de las diferencias de ingreso entre segmentos de la población urbana alargará las distancias sociales entre los pobres y los no pobres.

La conversión de las disparidades de ingreso en disparidades sociales tiene ciertamente muchas explicaciones. Quizás la más simple es aquella que sostiene que, conforme los hogares favorecidos se alejan de la mediana de ingresos de la población, aumenta su propensión a adquirir en el mercado servicios de mejor calidad que los colectivos. Cuando esta conducta se extiende a prestaciones básicas como el transporte, la educación, la seguridad pública, la salud y los servicios de esparcimiento, se producen al menos tres cambios importantes en la estructura social, los cuales alimentan a su vez los mecanismos de aislamiento social de los pobres urbanos. En primer lugar, se reducen los ámbitos de sociabilidad informal entre las clases a que da lugar el uso de los mismos servicios. Segundo, también se encoge el dominio de problemas comunes que los hogares enfrentan en su realidad cotidiana. Tercero, los servicios públicos pierden el importante sostén que se derivaba del interés de los estratos medios (donde se concentran los que tienen "voz") por mantener la calidad de las prestaciones que utilizaban, activando de ese modo un círculo vicioso de diferencias crecientes de calidad entre los servicios públicos y los privados, lo cual tiende a deteriorar aun más la posición de los pobres con respecto al resto de la sociedad.

Las disparidades de ingresos y las diferencias en cuanto a protecciones y estabilidad laboral también se manifiestan en la localización de las clases en el territorio urbano. De hecho, y como discutiré más adelante, una de las expresiones más notorias de la reducción de los ámbitos de interacción informales entre distintos estratos socioeconómicos es la progresiva polarización en la composición social de los vecindarios.
El resultado de estos procesos es un creciente aislamiento social de los pobres urbanos con respecto a las corrientes principales de la sociedad. Dicho aislamiento se convierte en un obstáculo importante para acumular los activos que se necesitan para dejar de ser pobre, lo que hace que la pobreza urbana socialmente aislada se constituya en el caso paradigmático de la exclusión social.

En lo que sigue examinaré algunos de los procesos más importantes que se aúnan en la actualidad para producir los tres cambios en la estructura social antes mencionados. Me refiero al aumento de la proporción de la población económicamente activa que muestra un vínculo precario e inestable con el mundo del trabajo; a la progresiva reducción de los espacios públicos que posibilitan el establecimiento de contactos informales entre las clases en condiciones de igualdad, y a la creciente concentración de los pobres en espacios urbanos segregados. El primer proceso guarda relación con el aumento de la precariedad e inestabilidad como rasgo de los mercados laborales; el segundo, con la segmentación de los servicios - principalmente de la educación-, y el tercero, con la segregación residencial. En el recuadro 1 se resumen las principales hipótesis de trabajo concernientes a cada uno de estos campos.

Para simplificar la presentación, el recuadro 1 no hace referencia a otros servicios básicos, pero ciertamente la salud, el transporte, la seguridad pública y los lugares de recreación y esparcimiento colectivo, entre otros, delimitan espacios de interacción con mecanismos específicos de integración y de segmentación. Con sus matices particulares, el funcionamiento de cada uno de ellos va configurando el escenario de estructuras de oportunidades donde se nutre el portafolio de activos de los pobres urbanos, contribuyendo de ese modo a definir su localización en la estructura social de la ciudad.

En lo que sigue comentaré con más detalle la naturaleza, los factores determinantes y las consecuencias de las segmentaciones en el ámbito laboral, educativo y residencial. ${ }^{4}$ nocer que la evidencia acumulada en los países desarrollados tiende a mostrar que las diferencias de regímenes de bienestar, si bien atenúan los impactos de la globalización sobre los hogares con menos activos sociales, no bastan para modificar la dirección de las tendencias. Como señala Esping-Andersen (1999), la preservación de distribuciones más equitativas del ingreso suele hacerse a costa de altas tasas de desempleo, y allí donde éstas son bajas, aumentan las inequidades. De tales constataciones surge la sospecha de que la tensión entre equidad y pleno empleo puede ser un fenómeno inherente al despliegue del nuevo estilo de crecimiento económico.

\footnotetext{
${ }^{4}$ En diferentes partes del texto utilizo los términos "diferenciación", "segmentación" y "segregación", por lo que conviene aclarar el significado que asigno a cada uno de ellos. El primer término simplemente designa diferencias en los atributos de dos o más categorías sociales. El segundo agrega al anterior una referencia a la existencia de barreras para el paso de una categoría a otra. El tercero agrega a los dos anteriores una referencia a la voluntad de los miembros de una u otra categoría de mantener o elevar las barreras que las separan entre sí.
} 
Recuadro 1

Posible INCIDENCIA DE SEGMENTACIONES EN ÁREAS DE LA ESTRUCTURA SOCIAL SOBRE LA FORMACIÓN DE ACTIVOS QUE PODRÍAN AMORTIGUAR LAS TENDENCIAS AL AISLAMIENTO DE LOS POBRES URBANOS

\begin{tabular}{ll}
\hline Segmentaciones & Capital social individual \\
\hline En el área laboral. & $\begin{array}{l}\text { Reduce la probabilidad } \\
\text { de contar con redes de in- } \\
\text { formación y contactos } \\
\text { que facilitan la búsqueda } \\
\text { de empleo. }\end{array}$
\end{tabular}

En el área educativa.

\begin{abstract}
Se debilitan i) la formación de reciprocidad y solidaridad; ii) la posibilidad de que los que tienen más conozcan los méritos de los que tienen menos y construyan lealtades con ellos; iii) la posibilidad de incorporar hábitos y actitudes de clase media con respecto a la movilidad, por medio de la educación.
\end{abstract}

En las áreas residen- Menor información y ciales.
Menor eficiencia normativa.

Menor exposición a modelos de rol.

\section{Capital social colectivo Capital ciudadano}

La separación de los lugares de trabajo que reclutan a los que tienen "voz" reduce la fortaleza de las instituciones laborales y de las reivindicaciones que pueden articular los pobres urbanos.

Se reduce la participación de los padres de estudiantes de clase media en la educación pública y se atenúa su influjo sobre el mantenimiento de la calidad de estos servicios.

Riesgo de declinación de las instituciones vecinales por déficit de liderazgos.
El trabajo deja de operar como el vínculo central de pertenencia a la sociedad. Se afecta la adquisición de derecho ciudadano. Se debilitan los sentimientos de ciudadanía al no compartir problemas y destinos con las corrientes predominantes de trabajadores.

Los estudiantes pobres ven reducidas sus oportunidades de experimentar la pertenencia a una comunidad con iguales derechos y obligaciones, problemas similares y recompensas por méritos con sus pares de otras clases.

Debilitamiento de los sentimiento de ciudadanía al no compartir problemas vecinales con otras clases, y riesgo de formación de subculturas marginales.

\section{II}

\section{La segmentación laboral}

Para los efectos del análisis que se desarrolla enseguida, conviene comenzar recordando las observaciones sintetizadas en el recuadro 1 sobre ciertos aspectos del portafolio de activos de los pobres urbanos que podrían verse afectados por las transformaciones en el mercado laboral y que tienen incidencia en su grado de aislamiento o de integración en la sociedad: i) Dimensión de capital social individual: el establecimiento donde se trabaja es un lugar privilegiado para la construcción de redes de amistad, a través de las cuales fluyen recursos en forma de contactos, información y facilidades de acceso a determinados servicios.

ii) Dimensión de ciudadanía en sus aspectos subjetivo y objetivo: es también un ámbito privilegiado 
para la generación de elementos subjetivos de ciudadanía, en el cual se comparten problemas, se consolidan identidades, se afianzan autoestimas y se construye un destino común. Pero también lo es para la adquisición de derechos objetivos de ciudadanía, por medio de conquistas laborales tales como la ampliación y el mejoramiento de las prestaciones sociales usualmente asociadas al rol de trabajador asalariado.

iii) Dimensión de capital social colectivo: la participación estable en un mismo establecimiento de trabajadores con distinto grado de calificación aumenta las oportunidades que tienen las categorías de trabajadores menos calificados de acceder a instituciones eficientes en la defensa de sus intereses laborales y en la preservación de derechos ya adquiridos.

Una de las características nodales de la presente reestructuración económica radica en que los umbrales de calificación para participar en el mercado formal se elevan al ritmo cada vez más acelerado de las innovaciones tecnológicas y de los requerimientos de productividad y competitividad a nivel mundial. Esta situación suele implicar una drástica devaluación de los créditos asociados a las habilidades y competencias que los trabajadores lograron adquirir en los lugares de trabajo y, por ende, una reducción igualmente drástica de sus posibilidades de participar en el mercado formal y en los ámbitos laborales donde se acumula el tipo de activos antes mencionados. Paralelamente se producen intensos procesos de desindustrialización y de achicamiento del Estado, con la consecuente reducción de la proporción de puestos de trabajo estables y protegidos, y el consiguiente aumento de los servicios, particularmente de los personales y los de consumo. Ahora bien, como en los servicios prevalece una distribución del ingreso y de las calificaciones más polarizada que en la industria, la transferencia masiva de mano de obra de un sector a otro se vincula a un incremento de la desigualdad en los ingresos y en las condiciones de trabajo. ${ }^{5}$ Por otra parte, muchas de las actividades de servicios personales y de consumo pueden verse como extensiones de las tareas domésticas,

\footnotetext{
5 Existe un amplísimo debate sobre los efectos de la desindustrialización en la desigualdad (véase, entre otros, Sassen, 1999; Mollenkopf y Castells, 1991; Hamnett, 1998; Musterd y Ostendorf, eds., 1998). Uno de los ejes de ese debate es la falta de consideración de procesos que pueden estar incidiendo en el aumento de la desigualdad de ingresos y que tienen que ver, entre otras cosas, con cambios en los sistemas impositivos, en los beneficios sociales, en el desempleo, en la composición de los hogares o en la estructura de edad de la población. En el centro de estos cuestionamientos se encuentra el análisis de la acción del Estado.
}

por lo cual ofrecen márgenes muy estrechos para aumentar la productividad. Como argumenta EspingAndersen (1999), citando a Baumol (1967), estas dos características exponen a estas actividades a una "enfermedad de costos" (cost disease) que las hace intrínsecamente precarias e inestables.

Para apreciar cuán importantes son estos procesos para los trabajadores latinoamericanos basta tener presente dos hechos. El primero es que la actual tendencia a la mayor precariedad del empleo se da en sociedades en las que ya se ha pasado del predominio de interacciones reguladas por una "solidaridad mecánica" al predominio de una "solidaridad orgánica" (Durkheim, 1964). Esto quiere decir que con la mayor división del trabajo, y bajo el impulso de la necesidad de interdependencia que generan los procesos de diferenciación y especialización, el eje de la integración en la sociedad se fue trasladando desde las instituciones primordiales (familia y comunidad, principalmente) al mundo laboral, lo que permitió abrigar la esperanza de que, al igual que en las naciones de industrialización temprana, el trabajo se constituiría en la vía privilegiada para la integración en la sociedad y la formación de identidades y sentimientos de autoestima.

Sin embargo, esa promesa parece ir perdiendo actualidad para la masa creciente de población que, en el nuevo contexto económico global, no logra establecer con el mercado de trabajo vínculos suficientemente estables y protegidos como para servir de plataforma a procesos de integración social. Para estos grupos, el trabajo deja de constituir la principal actividad sobre la que se apoya la estructuración racional de la vida cotidiana. Se debilita el rol del trabajo como articulador de identidades, como generador de solidaridades en la comunidad laboral y en las instituciones que de allí derivan y, en la medida en que la reducción de las formas estables de participación en el mercado y el debilitamiento de sus organizaciones cierran fuentes importantes de construcción de derechos, pierde también relevancia como promotor de ciudadanía.

El segundo hecho vinculado a las consecuencias de la mayor precariedad e inestabilidad laborales sobre las condiciones de vida de los pobres urbanos tiene que ver con el tipo de régimen de bienestar predominante en la región. ${ }^{6}$ Aun cuando en ninguno de los países de América Latina las prestaciones sociales han

\footnotetext{
${ }^{6}$ Por régimen de bienestar entiendo el conjunto más o menos articulado de protecciones ante riesgos sociales que brindan las instituciones del Estado, del mercado, de las familias y de la comunidad (Esping-Andersen, 1999).
} 
alcanzado un nivel de cobertura, calidad y articulación que amerite incorporarlas a la categoría de "regímenes de bienestar" en el sentido que les da Esping-Andersen (1990 y 1999), los embrionarios regímenes que se establecieron en la región siguieron moldes que se acercan más al modelo "conservador" de Europa continental, con énfasis en la asignación de derechos por medio del trabajo, que al modelo "socialdemocrático" de los países nórdicos, que apunta a derechos universales de ciudadanía, o al modelo liberal de los países anglosajones, con su foco en la provisión de redes de seguridad para los pobres y marginales. En este sentido, la institucionalidad regional que tiene que ver con la socialización de los riesgos está escasamente preparada para proteger a la población que tiene vínculos precarios e inestables con el mercado de trabajo.

Ciertamente, el Estado desempeña un papel medular en la determinación de los efectos de la reestructuración económica sobre la segmentación laboral. Un salario social garantizado reduce la compulsión de los individuos a aceptar trabajos poco atractivos, como son los de baja calificación en los servicios. Los programas públicos de empleo permiten absorber temporal- mente a los trabajadores desplazados por la tecnología en labores relacionadas con el funcionamiento de distintos servicios. Los cambios en el sistema impositivo pueden activar fuentes potenciales de trabajo. En general, el Estado puede dosificar e ir equilibrando la cobertura y el volumen de los recursos que transfiere a las categorías sociales más afectadas por las reformas económicas, dándole un tono más o menos progresivo a su acción y reflejando una mayor o menor voluntad de amortiguar los efectos concentradores de aquéllas. La consideración de estos factores ayuda a comprender las diferencias que se dan entre países desarrollados con distintos regímenes de bienestar, en lo referente a los cambios en la distribución del ingreso y en el peso de las actividades informales. Hay que tener presente, sin embargo, que aun los estudios que subrayan las diferencias entre los regímenes reconocen que, bajo las presiones derivadas tanto de la ampliación de las fronteras de competitividad como de los cambios en las estructuras demográficas, se observan indicios de repliegue de la cobertura de seguridad social incluso en aquellos países que se habían distinguido por sus avances en este campo. ${ }^{7}$

\section{III}

\section{La segmentación educativa}

La creciente centralidad del conocimiento como instrumento para el progreso de las naciones reafirma el papel que se asignó tradicionalmente a la educación como vía principal de movilidad social y ámbito privilegiado para la integración social de las nuevas generaciones. Ese papel ha sido reiteradamente destacado en los pronunciamientos de las cumbres presidenciales de los últimos años, donde los máximos responsables de las políticas públicas han reconocido que la equidad en los primeros años de vida debe formar parte del núcleo valorativo de los modelos que orientan el desarrollo en América Latina, y que la concentración de los recursos de los sistemas educativos en los niños de hogares con bajos niveles socioculturales es uno de los medios más eficientes para quebrar los mecanismos de reproducción de la pobreza y de la segmentación social.

Resulta paradójico, sin embargo, que junto con enunciar estos principios, muchas sociedades de la región estén experimentando un proceso históricamente inédito de estratificación de los circuitos educativos. ${ }^{8}$ Parece evidente que el sistema educativo mal puede estar habilitado para contribuir a levantar la hipoteca social de pobreza y desigualdad, y para contrapesar la creciente segmentación laboral, si la misma institución está segmentada. Ciertamente, éste es uno de los principales nudos del dilema social contemporáneo en muchos países latinoamericanos.

\footnotetext{
${ }^{7}$ Para el caso de Francia, véase White, 1998; para el de Alemania, Friedrichs, 1998; para el de Bélgica, Kestellot, 1998, y para el de Suecia, Borgegard, Anderson y Hjort, 1998.

${ }^{8}$ Tanto es así, que en algunos países de la región la conciencia de que existen tales circuitos hace que muchos padres vivan una etapa de gran ansiedad cuando intentan que sus niños de tres o cuatro años entren en determinado jardín infantil (donde hasta se les toma examen de ingreso), porque esa incorporación los habilitará posteriormente para continuar en un circuito educativo de escuelas y colegios con cuerpos docentes y equipamiento pedagógico de alta calidad, que a su vez les abrirán las puertas de las mejores universidades.
} 
Para entender mejor la importancia de la composición social de los lugares de enseñanza para la integración de las nuevas generaciones basta considerar el hecho de que aparte de los períodos de conscripción obligatoria en las fuerzas armadas (en los escasos países donde esta obligación está vigente y su aplicación es efectivamente universal), son muy pocas las instituciones de paso obligado para los ciudadanos que brindan a personas de distinto origen social la oportunidad de interactuar por tiempo prolongado sobre bases distintas al contrato de trabajo o al intercambio comercial de bienes y servicios. Sin duda, el sistema educativo es el principal -y muchas veces el únicoámbito institucional que tiene la potencialidad de actuar como un crisol integrador, según sea su capacidad para generar contextos en que niños y adolescentes pobres tengan la posibilidad de mantener una relación cotidiana con sus pares de otros estratos y desarrollar con ellos códigos comunes y vínculos de solidaridad y afecto bajo condiciones de igualdad.

Si los ricos van a colegios de ricos, si la clase media va a colegios de clase media y los pobres a colegios de pobres, parece claro que el sistema educativo poco puede hacer para promover la integración social y evitar la marginalidad, pese a sus esfuerzos por mejorar las oportunidades educativas de los que tienen menos recursos. ${ }^{9}$ Por ello es importante destacar no sólo la contribución que el sistema educativo hace a la equidad por medio de una mayor igualdad en las oportunidades de acceso, sino también su contribución a la integración de la sociedad, al crear las condiciones que facilitan la interacción entre desiguales en condiciones de igualdad.

\footnotetext{
${ }^{9}$ Cuando se examinan estos problemas, debe tenerse en cuenta que la distinción entre establecimientos pagados y no pagados, privados y públicos, no agota las formas que toma la segmentación educativa. Tanto la calidad de estos servicios como la homogeneidad en la composición social del público que atienden pueden y suelen acompañar a los procesos de segregación residencial, lo que hace que la asistencia a la escuela pública de los que más tienen no sea garantía de que el sistema educativo esté cumpliendo su función de integración social. Algunos arreglos político-administrativos tienen el mismo efecto, como en el caso de los condados de los Estados Unidos, en los que la autonomía de financiamiento de las municipalidades parece neutralizar totalmente el propósito integrador (Massey,1996), pues en la medida en que los que no pertenecen a una comunidad próspera no pueden utilizar sus servicios públicos, los resultados en términos de segregación de la sociedad pueden ser similares a los de la privatización de esos servicios.
}

Avanzando ahora sobre la síntesis presentada en el recuadro 1, cabe afirmar que el sistema educativo puede hacer una importante contribución a la equidad en la distribución de activos de capital social, al facilitar la construcción de redes de estudiantes de composición social heterogénea. Para los estudiantes pobres, esas redes son depósitos de reciprocidades, confianzas y lealtades que pueden ser activadas en el momento de su incorporación al mercado de trabajo, gracias a la movilización de los "créditos" acumulados con sus pares más influyentes a lo largo de una historia común, y gracias al conocimiento directo que éstos tienen de sus méritos (Flap y Graaf, 1986). Creer que únicamente los méritos van a ayudar a la movilidad social es una ficción que sólo se cumple en situaciones extraordinarias. Son los contactos sociales lo que potencia el aprovechamiento del capital humano $\mathrm{y}$, dado que generan una razonable certidumbre respecto al logro de empleos adecuados, lo que alimenta también la motivación para seguir invirtiendo en el desarrollo de ese capital.

De manera similar, y habida cuenta del impacto que suele tener la deserción del sistema de educación pública de sectores de clases medias que tienen "voz", toda iniciativa que impida el avance de la segmentación educativa hace, por una parte, una contribución indirecta al capital social colectivo de los estudiantes pobres, al frenar o contrarrestar el deterioro de la calidad de dicho sistema con respecto al privado. Y, por otra, ayuda al desarrollo temprano de sentimientos de ciudadanía entre los estudiantes pobres, que se nutren así de su participación, en condiciones de igualdad, en una comunidad educativa de la que forman parte sus pares de hogares más aventajados, de la que emergen identidades compartidas y metas comunes, actitudes positivas de reconocimiento del otro como sujeto de derechos, así como sentimientos de obligación moral que se extienden a compañeros de distinto origen social, religioso, étnico o nacional. En otras palabras, la contribución de la experiencia estudiantil a la formación ciudadana será más rica allí donde haya mayor semejanza entre la composición social de la comunidad escolar de cada establecimiento y la de la comunidad nacional. En cambio, conforme aumenta la segmentación entre los establecimientos educativos, aumenta también la probabilidad de que los miembros de un estrato social sólo se encuentren en una relación cara a cara con miembros de otros estratos sociales en el mercado de trabajo, donde las relaciones ya estarán enmarcadas en los patrones jerárquicos propios de la organización del mundo laboral. 


\section{IV}

\section{La segregación residencial}

La segregación residencial refiere al proceso por el cual la población de las ciudades se va localizando en espacios de composición social homogénea. Entre los factores más importantes que se invocan como antecedentes de estos procesos están el grado de urbanización y la urbanización de la pobreza, el grado de concentración de la distribución del ingreso, las características de la estructura de distancias sociales propias de cada sociedad y la homogeneidad o heterogeneidad de la composición étnica, religiosa o por origen nacional de la población de las ciudades.

Numerosos analistas de la situación imperante en los Estados Unidos, pero también en grandes centros urbanos europeos, han llamado la atención sobre la concentración de los pobres en determinados barrios de las ciudades. En esos barrios se concentra una densidad de privación material sin precedentes, que contrasta fuertemente con la concentración espacial igualmente inédita de hogares ricos en otros barrios. A juicio de estos analistas, debido al peso relativo de la población afectada, así como a la gravedad de las consecuencias que ello tiene sobre sus oportunidades de integrarse en la sociedad y sobre la salud del tejido social, las formas de segregación residencial que afectan a los pobres urbanos demandan una atención preferencial de los encargados de las políticas sociales (Massey, 1996; Wilson, 1987, y Wilson, ed., 1993).

Aun cuando aluden a una gama claramente insuficiente de factores determinantes, los intentos de dar cuenta de la segregación residencial, básicamente en términos de la urbanización de la pobreza y del aumento de la densidad urbana (Massey, 1996), resultan útiles para subrayar la importancia y la singularidad histórica de la concentración de los pobres en las ciudades como condición necesaria para la activación de los mecanismos que eventualmente pueden conducir a su aislamiento social. Una contribución adicional es la de proveer una explicación sencilla para la formación de subculturas que se apartan de las corrientes predominantes en la sociedad. De acuerdo con la teoría de Fischer (1975), la subcultura emerge en forma natural de la concentración espacial de categorías de población que comparten características similares, y el simple hecho de la mayor accesibilidad intragrupal favorece- ría la formación de patrones normativos que reflejan esos rasgos comunes. ${ }^{10}$

\section{Una tipología de barrios populares urbanos}

Ahora bien, se puede argumentar que los estratos populares de las grandes ciudades siempre se han aglutinado en vecindarios que se diferenciaban del resto por el nivel socioeconómico medio de sus habitantes, así como por una constelación de rasgos singulares propios de los patrones de interacción que se dan dentro del barrio y entre éste y el resto de la ciudad, constelación que, abusando ciertamente del término en muchos casos, podría estar indicando la presencia de una subcultura. Si esto es así, cabe preguntarse qué es lo que hace que el aislamiento social, a diferencia de otras experiencias de concentración espacial de estratos populares urbanos, se plantee actualmente como una característica central de las nuevas experiencias de segregación residencial de los pobres urbanos.

Para el solo efecto de facilitar la búsqueda de respuestas a este interrogante, en el recuadro 2 se presenta una tipología de barrios populares urbanos gruesamente caracterizados. Los casilleros fueron ordenados según un hipotético grado de apertura a la movilidad individual o colectiva prevaleciente en el período histórico en que se consolidan esos barrios. Como se infiere fácilmente de su lectura, en el recuadro se parte del supuesto de que el conocimiento de las transformaciones que experimentan las estructuras de oportunidades más importantes para los trabajadores no calificados y semicalificados es un antecedente esencial para comprender las distintas variedades de barrios pobres urbanos.

Sin embargo, antes de pasar al examen de cada una de las celdillas del recuadro 2 , debo advertir al lector sobre sus limitaciones en al menos dos aspectos importantes. En primer lugar, el esquema nace de re-

\footnotetext{
${ }^{10}$ En el caso de los guetos urbanos, tal como sostiene Crane (1989) en su estudio sobre la deserción escolar y la maternidad adolescente en Chicago, es probable que se sobrepasen los umbrales de densidad de la pobreza a partir de los cuales las pautas que se van consolidando alrededor de los correlatos conductuales de experiencias prolongadas de carencias críticas comienzan a operar como el marco dominante que orienta la acción.
} 
Recuadro 2

TIPOLOGÍA DE BARRIOS POPULARES URBANOS SEGÚN OPORTUNIDADES DE MOVILIDAD PREDOMINANTES DURANTE EL PROCESO DE SU FORMACIÓN

Individual

\begin{tabular}{lll}
\hline COLECTIVA & Favorable & Desfavorable \\
\hline
\end{tabular}

Favorable

Desfavorable
Barrios populares heterogéneos

(3)

Barrios de migrantes recientes

(1)
Barrios obreros tradicionales

Guetos urbanos

(4) flexiones acerca de la historia de las transformaciones urbanas en los países del Cono Sur. Como tal, es probable que sea útil también para interpretar la realidad de la morfología social urbana de las grandes ciudades de otros países de la región con características similares a las que sirvieron de base para su construcción. En cambio, su aplicabilidad es dudosa en aquellas sociedades latinoamericanas marcadas por heterogeneidades culturales que se basan en fuertes diferencias étnicas. En estos casos, la legitimidad de las pretensiones de superioridad social de las clases medias y altas no parece abiertamente cuestionada, y las distancias sociales muestran una solidez tal que no se ven afectadas por la proximidad o por la interacción cotidiana entre personas de distintas clases (el ejemplo arquetípico sería el de las castas de la India). En estos países, el problema de la segmentación en los espacios de interacción que controlan los distintos órdenes institucionales resulta secundario ante el desafío central que enfrentan los pobres urbanos, a saber, el de alcanzar los umbrales de acceso a la ciudadanía plena y legitimar sus pretensiones de integrarse en la sociedad en condiciones de igualdad.

Una segunda limitación del esquema es que tampoco considera los barrios formados mayoritaria o totalmente por minorías étnicas, religiosas, o por hogares que tienen un mismo origen territorial. Lo que distingue a tales barrios es el carácter voluntario de la decisión de instalarse en ellos. Los motivos pueden ser diversos: para restablecer redes, mantener costumbres, normas, valores e identidades culturales comunes; para defenderse de ataques de otros grupos sociales; para sentar las bases de proyectos empresariales en los cuales el capital social comunitario es un recurso muy valioso; para organizar, en condiciones más favorables, acciones colectivas tendientes a mejorar la infraestructura común o tendientes, incluso, a perseguir objetivos políticos de más largo plazo. Es interesante notar que en estos casos la segregación residencial puede ser un recurso instrumental deliberadamente buscado (Boal, 1998, p. 97), o una precondición para la formación de una comunidad, o un resultado de ello (Castles, 1998).

\section{a) Barrios de migrantes recientes (celdilla 1)}

Después de la Segunda Guerra Mundial, el crecimiento de este tipo de vecindarios en muchas ciudades de la región estuvo estrechamente ligado al ritmo de las transferencias masivas de población de origen rural, que en su mayoría se estableció en la periferia de los grandes centros urbanos.

Hay varios aspectos de la situación imperante en estos barrios que los diferencian de la situación de los pobres en los actuales guetos urbanos de la región. En primer lugar, muchos de los migrantes eligieron voluntariamente residir en la periferia de las ciudades, procurando la cercanía de familiares o de conocidos de igual o similar origen migratorio. Segundo, la mayoría de estas personas fueron atraídas por las posibilidades de movilidad social que ofrecía la ciudad. La conquista de una ciudadanía urbana representaba, entre otras cosas, acceso a servicios y a prestaciones sociales inexistentes en el lugar de origen. Tercero, el momento histórico en que se produjeron las migraciones permitió que germinaran expectativas de progreso sostenido. En efecto, la ampliación del aparato del Estado - paralela a la expansión de los servicios públicos - y la activación económica que acompañó el proceso de sustitución de exportaciones en las dos 
décadas posteriores a la Segunda Guerra Mundial generaron una capacidad de absorción de empleo que alcanzó a los trabajadores no calificados. Además, al comparar su situación presente con la de sus orígenes, muchos de los migrantes encontraban que sus esfuerzos estaban bien recompensados, lo que fue decantando un clima de optimismo y confianza en el progreso. Los propios actores, así como la mayoría de los analistas de estos procesos, percibían básicamente el paso por los cordones urbanos como una etapa intermedia en el proceso de asimilación a las ciudades. ${ }^{11}$

\section{b) Barrios obreros tradicionales (celdilla 2)}

Esta categoría se refiere a barrios en los cuales una importante porción de los residentes comparten experiencias de trabajo en los mismos establecimientos, como industrias, minas o empresas vinculadas al transporte. En muchas ciudades de América Latina se formaron barrios con esa configuración alrededor de astilleros, frigoríficos, talleres de ferrocarriles o establecimientos fabriles correspondientes a distintos sectores industriales. La característica distintiva de estas instancias era una conciencia de clase relativamente robusta, donde la sociabilidad del vecindario tendía a reforzar el microcosmos social que surgía alrededor del trabajo. ${ }^{12}$

${ }^{11}$ Sin duda esta rápida caracterización no hace justicia a la variedad de situaciones asociadas a los asentamientos urbanos de migrantes rurales en los distintos países de la región. A fines de la década de 1960 hubo un extenso y rico debate al respecto, en que participaron, entre otros, Roger Vekemans, José Nun, Fernando Henrique Cardoso, Aníbal Quijano y Gino Germani. Un buen resumen de la polémica suscitada desde distintas perspectivas en torno a la naturaleza de esos fenómenos se encuentra en un número totalmente dedicado a la marginalidad urbana de la Revista latinoamericana de sociología (1969). Es interesante observar que esta situación no es muy diferente de la que surge de los análisis de los procesos de traslado masivo de población rural a las ciudades de los Estados Unidos en los años veinte; también se mencionan tendencias similares en los países europeos después de la Segunda Guerra Mundial (véase Esping-Andersen, 1999). Algunos estudios ya argumentaban que los problemas sociales que afectaban a quienes se instalaban en los barrios más pobres de la ciudad obedecían a circunstancias temporarias que se irían desvaneciendo en el camino al progreso (Park y Burguess, 1925). Sin embargo, incluso la situación de los guetos urbanos altamente segregados en los años cincuenta en los Estados Unidos difiere radicalmente de las situaciones actuales, en el sentido de que la gran mayoría de aquellos trabajadores no calificados tenían empleo, y ello constituía una experiencia central en su vida. La segregación residencial actual, en cambio, se presenta en medio de una fuerte declinación de las oportunidades ocupacionales y de las remuneraciones relativas de los trabajadores no calificados (véase Wilson, 1996a, p. 54).

12 Un estudio realizado en Chile alrededor de 1960 permite examinar algunas de las formas en que las características del mundo laboral y de la comunidad de residencia se refuerzan mutuamente. La población estudiada en este caso fueron los mineros de la industria carbonífera de Lota, una zona aislada y económicamente deprimida, y los obreros de la industria del acero de Huachipato (Di Tella y otros, 1966).
A esa robustez contribuían varios factores. En primer lugar, la estabilidad de la inserción en un mismo ámbito de trabajo y el tamaño de los establecimientos. ${ }^{13}$ Segundo, la vigencia de utopías portadoras de imágenes que resaltaban la importancia del papel del trabajador en la construcción de una nueva sociedad, más rica, más equitativa y más integrada. Tercero, la acumulación de conquistas laborales gracias al esfuerzo colectivo y los avances paralelos en la adquisición de derechos ciudadanos. Cuarto, la importancia y fortaleza de las instituciones de los trabajadores, y, quinto, la esperanza de un progreso impulsado por la dinámica industrial. Bajo estas circunstancias, las actitudes y valores que emergían de la comunidad laboral tuvieron gran incidencia en la formación de los patrones que regulaban las relaciones entre los vecinos de barrios obreros. Y a su vez, la sociabilidad entre los vecinos y la participación en las instituciones vecinales realimentaban esas actitudes y valores.

\section{c) Barrios populares heterogéneos (celdilla 3)}

En esta celdilla entran aquellos vecindarios que se constituyeron en contextos urbanos que favorecían la movilidad individual y colectiva. Este fenómeno tuvo vigencia sólo en algunas de las grandes ciudades de América Latina, y su peso relativo estuvo en relación directa con la antigüedad y profundidad de los procesos de industrialización y urbanización. En ellos convivían, entre otros, obreros industriales estables, otros que habían alcanzado la independencia mediante el establecimiento de talleres o pequeños comercios, trabajadores de servicios personales diversos, empleados de oficina o maestros, todos los cuales mantenían contactos informales cotidianos, donde unos y otros eran reconocidos fundamentalmente como buenos o malos vecinos, como personas decentes o no decentes, y donde importaban poco otras distinciones vinculadas a la condición socioeconómica de cada hogar. Aunque muchos de estos hogares bordeaban las fronteras de la pobreza, en conjunto reunían suficiente capacidad de consumo como para estimular el establecimiento de múltiples microempresas —y la residencia en el barrio de sus dueños - que brindaban una amplia gama de servicios, tales como comercios de todo tipo, peluquerías, cines, bares o talleres de reparación.

\footnotetext{
13 Lipset, Trow y Coleman (1962), en su investigación sobre la International Typographical Union, encontraron que había una relación entre el tamaño del establecimiento y el vigor de los lazos de amistad que se establecen, lazos que comprometen a su vez un rango amplio de valores, actitudes y actividades fuera del contexto donde se originó la interacción.
} 


\section{d) Guetos urbanos (celdilla 4)}

La celdilla 4 es principalmente el resultado de procesos de segregación residencial, que en América Latina han operado fundamentalmente a partir de los años ochenta, en un contexto que muestra importantes diferencias con los procesos que caracterizaron la constitución de barrios formados por los nuevos obreros (migrantes internos) y los viejos obreros de las ciudades. Lo que prima en ese contexto son experiencias de desindustrialización y de achicamiento del Estado - es decir, el debilitamiento de dos de las fuentes más importantes de empleo urbano no precario-, de acelerado estrechamiento de las oportunidades laborales para trabajadores no calificados o semicalificados, y de rápida elevación de los umbrales de calificación requeridos para incorporarse en el mercado laboral. En vez de la atracción de la ciudad, opera la expulsión hacia la periferia. En lugar de estímulos nacidos de nuevas oportunidades de trabajo, crece la proporción de la población activa con pocas esperanzas de inserción estable en la estructura productiva. A diferencia de los migrantes rurales que comparaban favorablemente su situación presente con la que habían dejado, muchos de los actuales pobres urbanos ya han incorporado expectativas de ciudadanía plena en lo que concierne a derechos sociales, civiles y políticos, entre ellas aspiraciones legítimas de participar en los estilos de vida predominantes en la ciudad. En vez de expectativas de movilidad ascendente, prima entonces el reconocimiento de una movilidad descendente inevitable o de la imposibilidad de progresar. Los efectos negativos de todos estos procesos sobre el bienestar de los pobres urbanos y sus posibilidades de integración social se ven agravados por la combinación perversa de dos fenómenos: el eje de la formación de identidades se desplaza desde el mundo del trabajo al mundo del consumo, al tiempo que se amplía la brecha entre la participación material y la participación simbólica de estos estratos.

La concentración espacial históricamente inédita de personas con aspiraciones propias de la vida urbana, con graves privaciones materiales y escasas esperanzas de alcanzar logros significativos merced al empleo, suscita fuertes sentimientos de privación relativa. Bajo estas circunstancias, los nuevos guetos urbanos favorecen la germinación de los elementos más disruptivos de la pobreza. Los hogares que cuentan con recursos para alejarse de esos vecindarios lo hacen, lo que va dejando en el lugar una población residual, que vive en condiciones cada vez más precarias y se halla crecientemente distanciada de las per- sonas que reúnen los rasgos mínimos para tener éxito en la sociedad contemporánea.

La concentración espacial de personas que comparten estas características refuerza la precariedad del grupo por varias vías. En primer lugar, la interacción con los vecinos está limitada a personas cuyas habilidades, hábitos y estilos de vida no promueven resultados exitosos de acuerdo con los criterios predominantes en la sociedad. Segundo, las redes vecinales son ineficaces para la obtención de empleo o de información sobre empleo y oportunidades de capacitación. Tercero, la misma inestabilidad laboral genera dificultades para el mantenimiento de instituciones vecinales básicas y de niveles adecuados de organización y control social informal. Cuarto, los niños y jóvenes carecen de contactos con modelos de rol exitosos dentro de las corrientes principales de la sociedad, así como de oportunidades de exposición a esos modelos. Por último, las situaciones de desempleo persistente aumentan la predisposición a explorar fuentes ilegítimas de ingreso. Aun cuando en abstracto la comunidad local rechace esos comportamientos, en los hechos, la experiencia compartida de las penurias que impone la supervivencia cotidiana en esas condiciones da origen, por efecto de una mayor comprensión de sus causas, a una mayor tolerancia hacia esas desviaciones. La sedimentación progresiva de estas respuestas adaptativas va alejando la normatividad y los códigos imperantes en estos barrios de aquellos que predominan en el resto de la ciudad, acentuando de ese modo su aislamiento social.

\section{Auges, decadencias y transformaciones}

Es muy probable que una mirada atenta a la composición social de los vecindarios pueda descubrir en todo momento la coexistencia de barrios cuya composición los acerca a uno u otro de los tipos señalados, configurando un escenario en que se proyecta espacialmente la heterogeneidad de la pobreza. Lo que cambia es el peso relativo de cada una de estas categorías, por cuanto su crecimiento corresponde a distintas combinaciones de modalidades de desarrollo, grados de urbanización y tipos de regímenes de bienestar. De hecho, cada vecindario cambia su composición con mayor o menor velocidad, pero de manera continua, como resultado de hogares que se van y de otros que llegan, de unidades económicas que desaparecen y de nuevos emprendimientos que se establecen en la zona, de modo que, en cada período, la fotografía de cualquier barrio con cierta antigüedad revelaría residuos de distintas 
etapas de su formación, huellas de distintos modos de ordenar y ocupar el territorio, de los tipos de familia que prevalecieron, de actividades económicas que ya no operan o agonizan, así como de sucesivas configuraciones de estructuras sociales que dominaron la sociabilidad de su tiempo y le dieron su tono.

En suma, de la discusión anterior se desprenden al menos tres hipótesis de trabajo, cuya puesta a prueba podría permitir avances interesantes en la comprensión de los procesos de segregación residencial y su vinculación con el aislamiento social. La primera hipótesis plantea que la heterogeneidad de la pobreza se proyecta en el espacio urbano, lo que supondría una tendencia entre los hogares pobres a agruparse según calidades similares de sus portafolios de activos. La segunda, que subyace como supuesto a todo lo largo del análisis anterior, afirma que el nuevo escenario económico y social crea condiciones que favorecen el crecimiento de vecindarios que se acercan mucho, por sus características, a los rasgos típicos del gueto urbano, esto es, vecindarios donde permanecen los que no tienen recursos para instalarse en otra parte, se van los que pueden, y se suman los que son expulsados de otras áreas de la ciudad. La tercera sostiene que los hogares de este último tipo de vecindario muestran los niveles más altos de aislamiento social, esto es, los vínculos más débiles con el mercado, con los distintos servicios del Estado y con los segmentos de población urbana que orientan su comportamiento por los patrones normativos y valorativos dominantes.

\section{V}

\section{Las segmentaciones y el aislamiento social}

Aun cuando la afirmación deba ponerse a prueba en cada caso específico, es muy probable que, tal como se presenta en el gráfico 1, los tres tipos de segmentación recién examinados se potencien mutuamente en sus efectos en cuanto al aislamiento progresivo de los pobres urbanos. Así, el aumento de las disparidades en los ingresos y en las condiciones de trabajo que resulta del funcionamiento actual de la economía tendería a manifestarse en segmentaciones de los servicios y polarizaciones en la distribución de las clases en el espacio urbano, mientras que las formaciones subculturales que suelen acompañar la consolidación de la segregación residencial de los pobres reforzarían a su vez los procesos de diferenciación de ingresos y de segmentación de los servicios. A medida que se profundizaran las disparidades entre barrios socialmente homogéneos, éstas se irían manifestando en diferencias de calidad en la infraestructura de servicios, educación, salud, transporte, seguridad pública y espacios de esparcimiento y recreación, todo lo cual aumentaría el aislamiento social de los pobres urbanos y reduciría sus posibilidades de insertarse en forma estable y no precaria en el mercado de trabajo.

Pese a esta hipotética interdependencia entre los grados de segmentación en uno y otro de los ámbitos potenciales de interacción antes discutidos, el examen separado de cada uno de ellos tiene al menos dos virtudes. La primera es justamente plantear estas supues-

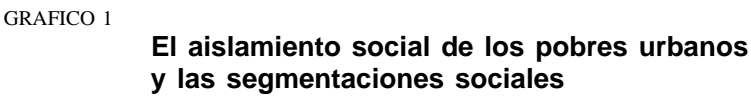

El aislamiento social de los pobres urbanos y las segmentaciones sociales

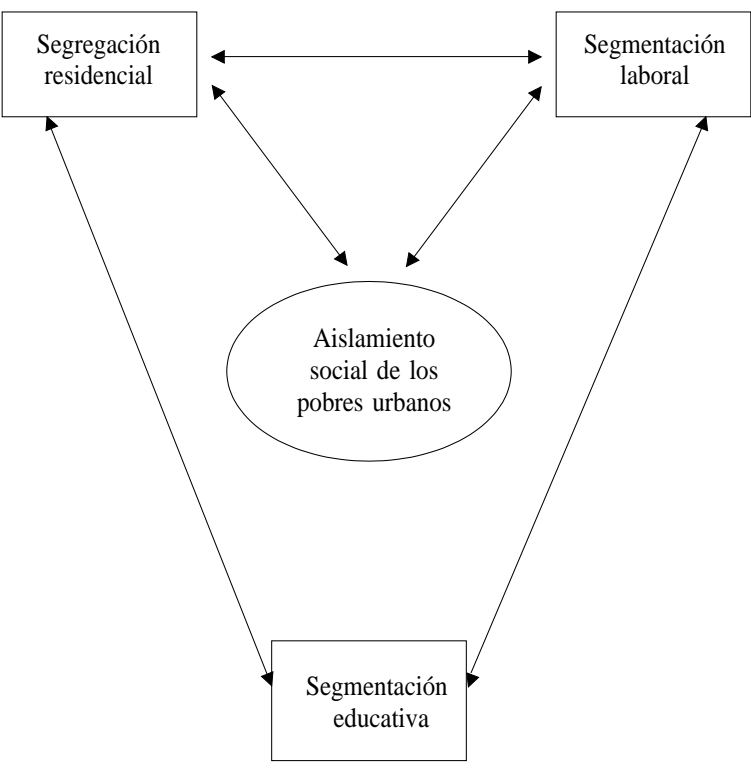

Activos afectados

- Capital social individual

- Capital social colectivo

- Capital ciudadano

tas interdependencias como hipótesis, incentivando de ese modo la identificación de los mecanismos que in- 
tervienen en la propagación de los efectos segmentadores de un ámbito a otro. La segunda es la de desplegar la batería de instrumentos de que dispondría el Estado para enfrentar los problemas de integración de los pobres urbanos, lo que le permitiría contar con una gama de alternativas para bloquear esos mecanismos de transmisión, de modo de reducir, frenar o contrarrestar las segmentaciones en los restantes ámbitos.

En efecto, dependiendo del carácter de las matrices socioculturales nacionales y de las concepciones de ciudadanía que guían las acciones de los Estados, el aumento de la segmentación y de la precariedad del mercado laboral puede coexistir con políticas que amortigüen su impacto sobre los diferenciales de ingreso o eviten la manifestación territorial de esas disparidades en términos de segregación residencial. El Estado también puede incentivar o desincentivar la universalidad en el uso de servicios básicos como el transporte, la seguridad pública, la salud y la educación, haciendo mayores o menores esfuerzos por mantener su calidad y dejando más o menos librada al juego de la oferta y la demanda la posibilidad de adquirir esos servicios en el mercado, opciones que tienen obvias implicaciones sobre la probabilidad de deserción de las clases medias y altas del ámbito público. Esta parece ser la postura asumida por algunos regímenes socialdemócratas de bienestar. En el caso de Suecia, por ejemplo, algunos autores afirman que si bien la creciente polarización social y económica entre vecindarios, que empezó a manifestarse desde comienzos de los años noventa, debilitó los espacios de interacción entre las clases, sus efectos sobre el aislamiento social de los pobres parecen haber sido adecuadamente contrarrestados por un Estado de bienestar que, aunque aquejado por problemas financieros, sigue manteniendo una cobertura generalizada de servicios que brindan protección y seguridad y que abarcan a todas las clases sociales (véase Borgegard, Anderson y Hjort, 1998).

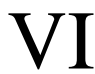

\section{Algunos mecanismos que retroalimentan el aislamiento social de los pobres}

\section{La independencia creciente de los efectos de las subculturas marginales sobre el comporta- miento de los pobres urbanos aislados}

Cualesquiera que sean sus causas, una vez que se instalan concentraciones de pobres involuntariamente aislados de las corrientes principales de la sociedad, se crean condiciones fértiles para la emergencia y perpetuación de subculturas marginales, toda vez que las reacciones que despiertan en el resto de la sociedad las conductas orientadas y reguladas por esas subculturas alimentan y profundizan el aislamiento social de los pobres urbanos.

Los ejemplos de la operación de estos círculos viciosos de reproducción ampliada del aislamiento social son muchos, y la mayoría de ellos se activa una vez que la opinión pública estigmatiza esos barrios como el espacio donde se congregan las "clases peligrosas". Basta mencionar tres de los ejemplos más conocidos: i) sus habitantes, especialmente los jóvenes, suelen ser víctimas de la llamada "discriminación estadística", en virtud de la cual la sola consideración de su lugar de residencia es suficiente para que algunos empleadores rechacen sus postulaciones de trabajo; ii) los hogares que pueden hacerlo desertan a otros barrios, lo que priva al vecindario de posibles modelos de rol, de personas que tienen "voz" y que habrían podido oficiar de transmisores de los patrones normativos de la sociedad global y de contactos e informaciones útiles para la obtención de empleos, el acceso a servicios o ambas cosas a la vez; y iii) las personas evitan entrar en esos barrios, lo que hace que se reduzca la frecuencia con que algunos nuevos residentes entran en contacto con amistades y familiares que viven en otras zonas de la ciudad (véase al respecto Zaffaroni, 1999).

Las subculturas marginales están constituidas por una amplia gama de patrones conductuales y normativos que van sedimentando alrededor del reconocimiento de las adversidades que comparte una población con graves carencias materiales y condiciones precarias de vida, de las barreras a la movilidad social y de la necesidad de encontrar bases comunes para construir o reconstituir autoestimas seriamente dañadas por la experiencia de exclusión. Como comenté anteriormente, la 
falta de empleos formales y estables hace que el mundo del trabajo pierda, paulatina pero inexorablemente, su papel como referente central para la organización de la vida cotidiana, para la provisión de disciplinas y regularidades y para la articulación de expectativas y el escalonamiento de metas, al tiempo que el progresivo aislamiento tiende a hacer cada vez más difusas las señales (cuando las hay) que desde la sociedad global indican caminos accesibles a personas de baja calificación para alcanzar condiciones dignas de vida. Todo ello va aumentando la permeabilidad de los pobres urbanos aislados a otras propuestas normativas que surgen en el entorno inmediato, algunas de las cuales incorporan orientaciones que no rechazan transitar por caminos ilegales para alcanzar las esquivas metas del consumo, mientras que el aislamiento social inhibe la eficacia de eventuales iniciativas que podrían contrarrestar esas predisposiciones mediante la invocación de las normas y valores modales de la sociedad.

Profundizando un poco más en las consecuencias de estos procesos, se puede argumentar que el aislamiento contribuye a agotar el portafolio de activos de los pobres, en la medida en que afecta su capacidad de acumulación de capital social. Hay por lo menos tres razones para ello. En primer lugar, el aislamiento reduce las oportunidades de movilizar en beneficio propio la voluntad de personas que están en condiciones de suministrar trabajo o información y contactos sobre empleos, sobre oportunidades de capacitación y sobre estrategias para un mejor aprovechamiento de los servicios existentes, mientras que los recursos que circulan por las redes internas de los vecindarios segregados tienden a ser superfluos y poco eficaces. En segundo lugar, se reduce la exposición a modelos de rol, esto es, a individuos que por haber alcanzado buenos niveles de vida gracias a su dedicación, talento o disciplina, pueden constituir ejemplos positivos de asociación entre esfuerzos y logros, debilitando de ese modo el atractivo de los canales legítimos de movilidad social como vías para satisfacer las aspiraciones de consumo de los pobres. En tercer lugar, se restringen las ocasiones que permiten compartir con otras clases el tipo de experiencias cotidianas que alimentan y preservan la creencia en un destino colectivo común, experiencias sobre las cuales descansan los sentimientos de ciudadanía. La idea de ser portador de los mismos derechos y de gozar de los beneficios del principio universal de igualdad y libertad para la vida social puede verse como una ficción cuando el distanciamiento con respecto a las corrientes predominantes de la sociedad se vive todos los días.
Las subculturas marginales entre los pobres urbanos deben ser consideradas, entonces, como algo distinto de lo que Lewis ha llamado "la cultura de la pobreza", cuyo contenido se podría sintetizar en términos de un legado de valores y normas cuyo conocimiento ayuda a comprender la permanencia de la pobreza. Más bien, el acento en este caso está puesto en las subculturas marginales como reacción a condicionantes estructurales que provienen del funcionamiento del mercado, del Estado y de la sociedad, esto es, como uno de los resultados de la progresiva sedimentación de respuestas adaptativas frente a un cúmulo de factores negativos que confluyen en un medio precario y segregado.

Desde el punto de vista de quienes están interesados en construir salidas para estas situaciones, la pregunta más importante se plantea en torno a la capacidad de dichas subculturas para seguir influyendo en el comportamiento de los jóvenes una vez desaparecidas las principales causas que las originaron. Esto es, en qué medida un incremento significativo del tiro de la chimenea económica permitirá incorporar al mercado a jóvenes con muy baja calificación y que en la actualidad se encuentran encapsulados dentro de una subcultura que no cree en la asociación entre esfuerzos y logros a través del trabajo.

William J. Wilson es optimista con respecto a esta situación en los Estados Unidos, y sostiene que un cambio en las oportunidades laborales podría anular el efecto del medio social inmediato. Sin embargo, no se debe subestimar la hipoteca que representan las cadenas estructurales que transmiten los efectos de una generación a la siguiente y que parecerían ir reduciendo el portafolio de activos con que los pobres que viven en barrios segregados podrían enfrentar el desafío de aprovechar eventuales nuevas oportunidades ocupacionales.

En una revisión exhaustiva de la literatura estadounidense sobre los efectos de los vecindarios sobre una serie de comportamientos considerados de riesgo, Jencks y Mayer (1990) encontraron abundante evidencia acerca de los efectos de contextos barriales segregados y homogéneamente pobres sobre el rendimiento educativo, sobre las conductas adictivas y delictuosas y sobre la maternidad adolescente. Por otra parte, en un estudio realizado recientemente en Montevideo, el autor del presente documento puso a prueba el efecto de la composición social del vecindario sobre el rendimiento educativo, la maternidad adolescente y también sobre el éxito de los jóvenes en el mercado de trabajo, medido por sus ingresos horarios. Los resul- 
tados de ese estudio tienden a corroborar la importancia del grado de homogeneidad en la composición social del vecindario como factor determinante de la emergencia de comportamientos de riesgo y de logros en el mundo del trabajo. El componente de riesgo de los comportamientos analizados radica justamente en su capacidad para operar como barreras para la acumulación de los activos que son requeridos para aprovechar las oportunidades que se presentan en el mercado, en la sociedad y en el Estado (Kaztman, coord., 1999).

\section{La deserción de los espacios públicos por las clases medias globalizadas}

Las sociedades pueden distinguirse según el tipo de reglas distributivas cristalizadas en sus estructuras básicas (mercado, régimen de propiedad, Estado y otras). Esas reglas, que determinan profundamente las condiciones de vida de la población, se traducen en actitudes individuales de mayor o menor tolerancia a la desigualdad. Esas formas institucionales, así como sus correlatos en contenidos mentales, constituyen elementos nodales de la matriz sociocultural que caracteriza a cada sociedad.

La noción de tolerancia a la desigualdad ayuda a comprender la estabilidad de algunos indicadores de equidad o inequidad. ${ }^{14}$ Se trata de estructuras subterráneas que, ante incrementos en los indicadores de desigualdad que sobrepasan lo tolerable, se activan para impulsar acciones solidarias que tienden a restablecer el equilibrio. Estas acciones pueden comprender desde apoyos electorales o iniciativas orientadas a proteger a los más débiles y mantener la calidad de los servicios de cobertura universal hasta la disposición a pagar impuestos para apuntalar medidas redistributivas. ${ }^{15}$

\footnotetext{
${ }^{14}$ En un estudio que compara datos sobre distribución del ingreso en distintos países y diferentes períodos se sostiene que la desigualdad del ingreso es relativamente estable en los países y en el tiempo, en marcado contraste con el comportamiento de las tasas de crecimiento del producto interno bruto, que sí cambian con rapidez y se caracterizan por ser muy poco persistentes (Li, Squire y Zou, 1998).

${ }^{15}$ Como argumenta Barry, la disposición a pagar impuestos altos, al mismo tiempo que permite elevar la calidad de las prestaciones colectivas, reduce los recursos disponibles de los grupos de mayores ingresos para invertir en la adquisición privada de los servicios, todo lo cual desalienta su deserción de los espacios públicos (Barry, 1998, p. 23). Ciertamente, la contribución de las clases medias y altas al mantenimiento de los espacios públicos que posibilitan la interacción entre las clases no descansa solamente en su grado de aversión a la desigualdad. También interviene el temor a los efectos negativos que suelen acompañar el deterioro de la calidad de
}

La aversión a la desigualdad descansa en la capacidad de empatía de los más aventajados con respecto a los que tienen menos y en sentimientos de obligación moral hacia ellos. Estos contenidos mentales pierden vigencia si no se renuevan periódicamente por medio de contactos informales entre personas de distinta condición socioeconómica. Los sentimientos serán más fuertes cuanto más intensa y más frecuente sea la interacción. El espacio de estos encuentros es el ámbito público (el transporte, las plazas, las escuelas y hospitales, las canchas de fútbol, los bares, las playas, los espectáculos masivos, las calles y otros). Tanto la segregación residencial como la segmentación de los servicios reducen esos espacios, debilitando de esa manera la base estructural que sustenta la capacidad de empatía y los sentimientos de obligación moral, lo cual afecta a su vez los niveles de intolerancia a la desigualdad y resta eficacia a los mecanismos homeostáticos. ${ }^{16}$

Los niveles altos de intolerancia a la desigualdad también operan como mecanismos de autocontrol en el consumo de las clases medias y altas, especialmente de aquellos consumos que establecen distancias irritantes y fácilmente visibles con las otras clases. Esos controles entran en conflicto con las expectativas que despierta la exposición -inevitable en los procesos de globalización - a los estilos de vida de sus pares de los países desarrollados. En la medida en que los recursos requeridos para satisfacer las nuevas aspiraciones de consumo compiten con aquellos que exige la satisfacción de las demandas de los pobres, el distanciamiento entre los patrones de consumo de las clases irá acompañado de una pérdida de interés de las clases acomodadas por la situación y el destino de las menos aventajadas. ${ }^{17}$

Los mecanismos de solidaridad social suelen resistir el aislamiento de un pequeño sector de la sociedad que, por su riqueza, siempre ha recurrido a

\footnotetext{
vida de las mayorías y de los servicios públicos a los que acuden. Tales efectos son la inestabilidad política, el descenso de la legitimidad de las instituciones - $-\mathrm{y}$ la consecuente dificultad de las élites para movilizar la voluntad colectiva en apoyo a proyectos de cambio-y, cada vez más, las consecuencias de la inseguridad pública sobre la calidad de las condiciones de vida.

16 Se entiende por mecanismos homeostáticos un conjunto de fenómenos de autorregulación conducentes al mantenimiento de una relativa constancia en las propiedades y composición del medio interno de un organismo.

17 Los países pequeños y de gran homogeneidad cultural crean ámbitos de cercanía que tienden a inhibir el distanciamiento de las élites, toda vez que la comunidad tiene en tal caso una mayor capacidad para sancionar a los que se apartan demasiado de los hábitos y estilos de vida de las mayorías.
} 
alternativas privadas de provisión de servicios. En cambio, las rupturas en el tejido social comenzarán a ha- cerse visibles allí donde una masa importante de las clases medias deserte de los servicios públicos.

\section{VII}

\section{Consideraciones finales}

"Seducidos y abandonados", la metáfora contenida en el título de este documento, pretende destacar una de las peculiaridades de la composición de la actual pobreza urbana en muchos países de la región: la creciente proporción de hogares que habiendo incorporado expectativas en cuanto a la conquista de una ciudadanía plena por medio del trabajo, y habiendo desarrollado aspiraciones de consumo propias de la sociedad de su tiempo, ven progresivamente debilitados sus vínculos con las fuentes de los recursos que hacen posible alcanzar esas metas. Han sido seducidos por una sociedad moderna en que sólo pueden participar simbólicamente, no pudiendo superar por sus propios medios los obstáculos para alcanzar una participación material equivalente.

El enfoque con que se plantea el examen de la naturaleza y los factores determinantes de estos fenómenos, que puede ser llamado "estructural", se diferencia de otros modos de analizar la pobreza urbana por su énfasis en la localización de estas categorías en la estructura social de las ciudades, esto es, en la existencia y calidad de los vínculos que se establecen con las estructuras de oportunidades que controlan el Estado, el mercado y otros órdenes institucionales de la sociedad. Desde esta perspectiva importa conocer el funcionamiento de los ámbitos de interacción que pueden operar como fuente de los activos que se requieren para la integración social en cada etapa histórica, de las relaciones que se establecen entre esos ámbitos, pero también del comportamiento de otros actores sociales y, en particular, de las lógicas que subyacen las decisiones de las clases medias en cuanto a promover los servicios y espacios públicos o a desertar de ellos (la "voz" y la "salida" de Hirschman).

He hecho particular hincapié en la discusión de los problemas de segregación residencial, en parte por entender que se trata de una dimensión que ha sido descuidada en la agenda social y, en parte también, por considerar que los barrios con alta densidad de hogares con privaciones materiales y aspiraciones frustradas son campo fértil para la aparición de comporta- mientos de riesgo y subculturas marginales, cuya cristalización agrega obstáculos —muchas veces difíciles de superar o cuya superación supone un enorme costo para toda la sociedad - a la acumulación de activos y refuerza, de ese modo, las tendencias al aislamiento social de los pobres urbanos.

Dado el estado embrionario del enfoque, las principales sugerencias en esta etapa son de orden más bien teórico. Esto es, a partir del reconocimiento de que el estudio de los problemas de segregación residencial y de la segmentación de los servicios constituye una vía promisoria para avanzar en la comprensión de las barreras a la equidad social, se pueden señalar diversas áreas que requieren más reflexión, así como la necesidad de poner a prueba algunas de las hipótesis postuladas a lo largo del documento. Sin embargo, a partir de lo que se ha adelantado hasta ahora, es posible sugerir un conjunto de orientaciones generales para mejorar las políticas públicas encaminadas a atacar las bases de la producción y reproducción de la pobreza urbana.

En primer lugar, se puede afirmar que cualquiera que sea la forma que adquiera la segregación residencial en las ciudades, sus consecuencias sobre el aislamiento de los pobres urbanos parecen ser lo suficientemente importantes como para que los encargados de las políticas de ordenamiento territorial no dejen librado el proceso a fuerzas del mercado orientadas esencialmente por una lógica inmobiliaria, caso en el cual las desigualdades de ingreso en las ciudades tenderán a fragmentar el espacio urbano en vecindarios que concentrarán clases homogéneas y en el cual, a la vez, la polarización espacial de las clases actuará como un cemento de las desigualdades que impedirá un posterior repliegue hacia situaciones más equitativas.

A este respecto, y para identificar las mejores prácticas, conviene examinar en detalle las acciones llevadas a cabo en países que han dado prioridad a estos problemas en su agenda social, especialmente en aquellos regímenes de bienestar socialdemócratas que se han caracterizado por la eficacia de sus políticas de 
integración social. En Suecia, por ejemplo, aun cuando se advierte un aumento de los índices de concentración del ingreso, los efectos que ello ha tenido sobre la segregación residencial se han visto morigerados por políticas del gobierno tendientes a mezclar tipos diferentes de hogares en edificios integrados. Consecuencias similares tienen las iniciativas de igualar el alquiler de las casas del mismo nivel pero ubicadas en vecindarios diferentes (Borgegard, Anderson y Hjort, 1998). En general, las políticas de desmercantilización de las viviendas por medio del control de los subsidios a los alquileres, o de facilitación del acceso a la propiedad de los sectores de menores recursos, así como las iniciativas dirigidas a una mayor integración habitacional, pueden considerarse legítimamente como parte integral de las políticas de bienestar (Murie, 1998). Debe tenerse presente que el Estado también puede replegarse en esta área o, como en los casos de Chile o Sudáfrica, aplicar políticas deliberadamente diseñadas para promover la segregación residencial (Christopher, 1998; Portes, 1989).

Las presiones tendientes a reducir el déficit fiscal y equilibrar las finanzas públicas, asociadas a la ampliación de las fronteras de la competitividad, también constriñen las alternativas abiertas al Estado para frenar o contrarrestar los procesos de segregación residencial. La búsqueda de un equilibrio financiero y el consecuente apremio por maximizar el uso de los recursos públicos inclinan al Estado a concentrar sus esfuerzos de construcción de viviendas populares en terrenos urbanos o periféricos de bajo valor, o a legalizar la ocupación de tierras en que los pobres, en busca de soluciones propias para los problemas de vivienda, establecen asentamientos y presionan posteriormente para su regularización.

La lentitud con que se va procesando el decaimiento de los espacios pluriclasistas de sociabilidad informal en las ciudades hace que sus consecuencias sobre la integración social pasen muchas veces inadvertidas para las mayorías ciudadanas. Por ende, sus efectos son usualmente subestimados como factor de integración, como fuentes de renovación de las reservas de altruismo, solidaridad y de actitudes de aversión a la desigualdad. Al respecto, como dice Caldeira (1996), al igual que la noción de ciudadanía, la idea de una ciudad abierta a todos, aun cuando nunca ocurre en toda su extensión, opera como un ideal legitimador de las demandas de incorporación de los grupos excluidos.

Por otra parte, el objetivo de fortalecer la integración social en las ciudades mediante la promoción de los espacios públicos pluriclasistas puede parecer alejado de las posibilidades de las políticas estatales, en parte porque los recursos para ello suelen competir con los requeridos por otras prioridades de la agenda social de alivio de la pobreza. Ciertamente, esa percepción se ajusta a la realidad de muchas de las grandes ciudades de la región, donde la segregación residencial, la segmentación de los servicios y la deserción de las clases medias de los lugares públicos de sociabilidad informal pluriclasista están tan avanzadas que las posibilidades de frenar o de contrarrestar estos procesos en el corto o mediano plazo pueden parecer irreales. Con todo, estas circunstancias no deberían disuadir la acción sino estimularla, puesto que la alternativa es una agudización progresiva de la exclusión, con consecuencias imprevisibles para el orden social y la convivencia civilizada. De hecho, esas consecuencias irrumpen tarde o temprano, a veces en forma violenta, anómica e inesperada, a través de los correlatos socialmente disruptivos de una pobreza marginada por la concentración de privaciones y por su progresivo aislamiento de las pautas modales de la sociedad. La respuesta de las clases medias es apartarse de los lugares y servicios públicos ocupados por las "clases peligrosas", cuyos comportamientos, cultivados en el aislamiento y la precariedad generalizada, aparecen a las otras clases como exóticos y desviados. La deserción de las clases medias no hace más que acentuar el decaimiento de los espacios públicos, estrechando de ese modo el campo de experiencias que estimulan la capacidad de empatía con los sectores menos favorecidos y los sentimientos de obligación moral hacia ellos, y elevando, por ende, el umbral de tolerancia a la desigualdad. La experiencia acumulada sobre las consecuencias de descuidar estos problemas en las grandes ciudades puede resultar particularmente útil para el diseño de medidas preventivas en las ciudades de tamaño intermedio.

Como se señala en el texto, las políticas de utilización de los lugares públicos, las de prevención de la segmentación de los servicios básicos, así como las acciones que tienen que ver con el ordenamiento urbano, marcan la multiplicidad de caminos por los cuales es posible promover la sociabilidad pluriclasista y contrarrestar las poderosas tendencias a la privacidad y al aislamiento entre las clases. En muchos de esos ámbitos, más que políticas específicas, lo que se requiere es la incorporación de un matiz que debe estar siempre presente en cada uno de los programas sectoriales, en virtud del cual se da preferencia a todas aquellas iniciativas que conduzcan a una mayor frecuencia y calidad en la interacción entre desiguales. 
Hay un gran número de experiencias exitosas que pueden constituir una base de información muy valiosa para los países que se dispongan a enfrentar estos problemas. Son muchas las iniciativas de integración social en las ciudades de América del Norte y Europa que, o bien como acciones específicamente diseñadas para ese fin, o bien como matices presentes en la elaboración de políticas sectoriales, afectan las medidas de ordenamiento urbano, la selección de beneficiarios de conjuntos habitacionales subsidiados, la defensa de la calidad de los servicios públicos y la promoción de espacios que estimulen los contactos informales entre las clases. Su examen minucioso permitirá seleccionar aquellas que mejor se ajusten a los recursos y a las características singulares de cada sociedad.
Barry, B. (1998): Social Exclusion, Social Isolation and Distribution of Income, Case paper, $\mathrm{N}^{\circ}$ 12, Londres, London School of Economics.

Baumol, W. (1967): Macroeconomics of unbalanced growth: The anatomy of urban city, American Economic Review, vol. 57, $\mathrm{N}^{\circ}$ 3, Nashville, Tennessee, American Economic Association.

Boal, F.W. (1998): Exclusion and Inclusion: Segregation and deprivation in Belfast, S. Musterd y W. Ostendorf (eds.), Urban Segregation and the Welfare State, Londres, Routledge.

Borgegard, L.E., E. Anderson y S. Hjort (1998): The divided city? Socioeconomic change in Stockholm metropolitan area, 197094, S. Musterd y W. Ostendorf (eds.), Urban Segregation and the Welfare State, Londres, Routledge.

Caldeira, T, (1996): Fortified enclaves: The new urban segregation, Public Culture, vol. 8, $\mathrm{N}^{\circ}$ 2, Estados Unidos.

Castles, S. (1998): The process of integration of migrant communities, Population Distribution and Migration. Proceedings of the United Nations Expert Group Meeting on Population Distribution and Migration, Nueva York.

Crane, J. (1989): Effects of neighborhoods on dropping out of school and teenage childbearing, C. Jencks y P. Peterson (eds.), The urban underclass, Washington, D.C., Brookings Institution.

Christopher, A. (1998): Desegregation and disintegration in South African metropolis, S. Musterd y W. Ostendorf (eds.), Urban Segregation and the Welfare State, Londres, Routledge.

Di Tella, T. y otros (1966): Huachipato et Lota: étude sur la conscience ouvrière dans deux entreprises chiliennes, París, Centre National de la Recherche Scientifique.

Durkheim, E. (1964): The Division of Labor in Society, Nueva York, The Free Press.

Esping-Andersen, G. (1990): The Three Worlds of Welfare Capitalism, Princeton, Nueva Jersey, Princeton University Press. (1999): Social Foundations of Post Industrial Economies, Oxford, Reino Unido, Oxford University Press.

Fischer, C.S. (1975): Toward a subcultural theory of urbanism, American Journal of Sociology, vol. 80, $\mathrm{N}^{\circ}$ 6, Chicago, Illinois, The University of Chicago Press.

Flap, H. D. y N. D. Graaf (1986): Social capital and attained occupational status, The Netherland Journal of Sociology, $\mathrm{N}^{\circ} 22$, Amsterdam, Países Bajos.

Friedrichs, J. (1998): Social inequality, segregation and urban conflict: The case of Hamburg, S. Musterd y W. Ostendorf (eds.), Urban Segregation and the Welfare State, Londres, Routledge.

Granovetter, M. (1985): Economic action and social structure: The problem of embeddedness, American Journal of Sociology, vol. 91, No 3, Chicago, Illinois, The University of Chicago Press.

Hamnett, C. (1998): Social polarization, economic reestructuring and welfare state regimes, S. Musterd y W. Ostendorf (eds.), Urban Segregation and the Welfare State, Londres, Routledge.
Hirschman, A. (1970): Exit, Voice and Loyalty, Cambridge, Massachusetts, Harvard University Press.

Jencks, C. (1993): Rethinking Social Policy: Race, Poverty and the Underclass, Nueva York, First Harper Perennial Edition.

Jencks, C. y S. Mayer (1990): The social consequences of growing up in a poor neighborhood, L. Lynn y M. McGeary (eds.), Inner City Poverty in the United States, Washington, D.C., National Academy Press.

Kaztman, R. (1997): Marginalidad e integración social en Uruguay, Revista de la CEPAL, No 62, LC/G.1969-P, Santiago de Chile, Comisión Económica para América Latina y el Caribe (CEPAL).

Kaztman, R. (coord.) (1999): Activos y estructuras de oportunidades: estudios sobre las raíces de la vulnerabilidad social en Uruguay, Montevideo, Oficina de la CEPAL en Montevideo/Programa de las Naciones Unidas para el Desarrollo (PNUD).

Kaztman, R. y otros (1998): Vulnerabilidad, activos y exclusión social en Argentina y Uruguay, Documento de trabajo, $\mathrm{N}^{\circ}$ 107, Santiago de Chile, Organización Internacional del Trabajo (OIT).

Kaztman, R. (2001): El aislamiento social de los pobres urbanos: reflexiones sobre su naturaleza, determinantes y consecuencias, Buenos Aires, SIEMPRO/UNESCO.

Kestellot, C. (1998): The geography of deprivation in Brussels and local development strategies, S. Musterd y W. Ostendorf (eds.), Urban Segregation and the Welfare State, Londres, Routledge.

Li, H., L. Squire y H. F. Zou (1998): Explaining international and intertemporal variations in income inequality, The Economic Journal, vol. 108, Oxford, Reino Unido, Basil Blackwell.

Lipset, S. M., M. Trow y J. Coleman (1962): Union Democracy, Nueva York, Anchor Books, Doubleday and Co., Inc.

Massey, D. (1996): The age of extremes: Concentrated affluence and poverty in the twenty-first century, Demography, vol. 33, $\mathrm{N}^{\circ}$, Madison, Wisconsin, University of Wisconsin, Center for Demography and Ecology.

Mollenkopf, J. y M. Castells (1991): Dual City: Reestructuring New York, Nueva York, Russell Sage Foundation.

Murie, A. (1998): Segregation, exclusion and housing in the divided city, S. Musterd y W. Ostendorf (eds.), Urban Segregation and the Welfare State, Londres, Routledge.

Musterd, S. y W. Ostendorf (eds.) (1998): Urban Segregation and the Welfare State, Londres, Routledge.

Park, R. y E. Burguess (1925): The City, Chicago, Illinois, University of Chicago Press.

Portes, A. (1989): Latin American urbanization in the years of the crisis, Latin American Research Review, vol. XXIV, No 3, New Mexico, University of New Mexico.

Revista latinoamericana de sociología (1969): vol. 5, № 2, Buenos Aires, Instituto Torcuato Di Tella.

Rodríguez, J. (2000): Segregación residencial socioeconómica: ¿qué es?, ¿cómo se mide?, ¿qué está pasando?, ¿importa?, Santia- 
go de Chile, Centro Latinoamericano y Caribeño de Demografía (CELADE).

Sabatini, F. (1999): Tendencias de la segregación residencial urbana en Latinoamérica: reflexiones a partir del caso de Santiago de Chile, serie Azul, $N^{\circ}$ 29, Santiago de Chile, Pontificia Universidad Católica de Chile, Instituto de Estudios Urbanos.

Sarlo, B. (1994): Escenas de la vida posmoderna: intelectuales, arte y videocultura en la Argentina, Buenos Aires, Ariel.

Sassen, S. (1999): La ciudad global, Buenos Aires, Eudeba.

Tilly, C. (2000): La desigualdad persistente, Buenos Aires, Ediciones Manantial.

Torre, J. C. y E. Pastoriza (1997): Mar del Plata, un sueño de los argentinos, mimeo.

White, P. (1998): Ideologies, social exclusion and spatial segregation in Paris, S. Musterd y W. Ostendorf (eds.), Urban Segregation and the Welfare State, Londres, Routledge.
Wilson, W. (1987): The Truly Disadvantaged: The Inner City, The Underclass and Urban Policy, Chicago, Illinois, University of Chicago Press.

(1996a): When Work Disappears: The World of the New Urban Poor, Nueva York, Knopf.

(1996b): The age of extremes: Concentrated affluence and poverty in the twenty-first century, Demography, vol. $33, \mathrm{~N}^{\circ}$ 4, Madison, Wisconsin, Universidad de Wisconsin, Center for Demography and Ecology, noviembre.

Wilson, W. (ed.) (1993): The Ghetto Underclass: Social Science Perspectives, Newbury Park, California, Sage Publications.

Zaffaroni, C. (1999): Los recursos de las familias urbanas de bajos ingresos para enfrentar situaciones críticas, R. Kaztman (coord.), Activos y estructura de oportunidades: estudios sobre las raices de la vulnerabilidad social en Uruguay, Montevideo, Oficina de la CEPAL en Montevideo/Programa de las Naciones Unidas para el Desarrollo (PNUD). 